\title{
FAHPSort: A fuzzy extension of the AHPSort method
}

\author{
Jana Krejčí \\ Department of Industrial Engineering, University of Trento, \\ Via Sommarive 9, Povo 38123, Italy, \\ jana.krejci@unitn.it \\ Alessio Ishizaka \\ Center for Operational Research and Logistics (CORL), Portsmouth Business School, \\ University of Portsmouth, Portsmouth PO1 3DE, United Kingdom, \\ alessio.ishizaka@port.ac.uk
}

\begin{abstract}
Analytic Hierarchy process (AHP) is a powerful method belonging to the full aggregation family of multi-criteria decision-making methods based on pairwise comparisons of objects. Since the information about the problem is usually not complete in real decision-making problems, it is difficult to express precisely the preferences on pairs of compared objects. This problem has been handled in the literature by introducing fuzziness into AHP. However, neither AHP nor its fuzzy extensions can deal with sorting decision-making problems, which form a significant part of decision-making problems. This paper presents the FAHPSort method - a fuzzy extension of the AHPSort method, which is an adaptation of the AHP method for sorting decision-making problems. The FAHPSort method handles the vagueness in meaning of linguistic terms expressing the intensity of preference of one object over another one. Key properties of the FAHPSort method are described in the paper, and the method is illustrated in a decision-making problem.
\end{abstract}

Keywords: FAHPSort; AHPSort; Sorting; Fuzzy AHP; Fuzzy pairwise comparison matrix; Constrained fuzzy arithmetic.

\section{Introduction}

The Analytic Hierarchy Process (AHP), a multi-criteria decision-making method developed by Saaty in the 1970s (Ref. 44 ), has been widely used for ranking a finite set of alternatives and for choosing the best alternative from a finite set of alternatives. AHP is continuously being used to support high-impact decisions in important decision problems in various fields ranging from engineering and industry applications 
through social sciences applications to applications in the medical sector (see, e.g., the review articles Ref. 12,37,48,51 ). The original AHP method has also undergone a great methodological development (see, e.g., the review of the main developments after 2010 in Ref. 25 ). AHP has also been integrated with a wide range of other methods (shortly called integrated AHP) in order to increase its flexibility and wide applicability (see, e.g., the review articles Ref. 18,19 ).

However, neither AHP nor integrated AHP are suited for dealing with sorting problems, which aim to assign each alternative into one of the predefined ordered classes. Thus, in Ref. 21, AHP was adapted into AHPSort that is able to deal with sorting problems. AHPSort has already been successfully applied to environmental, business, and purchase decision problems (see, e.g., Ref. 16,38,39 ). AHPSort utilizes the strengths of the original AHP method and combines them with new features suitable for sorting problems. The main advantage of AHPSort over AHP is that it does not require pairwise comparisons of alternatives with respect to criteria. Namely, the alternatives are not compared with each other but only with the profiles representing the classes for each criterion. This is in particular convenient in problems with a large set of alternatives, where this approach leads to reducing the number of pairwise comparisons required from the decision maker. On the other hand, AHPSort in contrast to AHP requires additional information from decision makers regarding the classes. In particular, AHPSort requires that profiles representing the classes for each criterion are defined, which might be in some decision problems difficult for decision makers.

AHPSort as well as AHP cannot properly capture the vagueness of preference information provided by decision makers. In fact, both AHPSort and AHP use standard Saaty's scale of linguistic terms and integers $1-9$ that are supposed to model the meaning of these linguistic terms. It was argued in the literature that crisp numbers cannot model well the vagueness in meaning of linguistic terms and that fuzzy numbers should be used instead. Thus, fuzzy extension of AHP has become a very popular research subject in recent decades. There are many different approaches proposed in the literature for the fuzzy extension of AHP, see e.g. Ref. 3, 4, 6, 8, 13, 20,28, 29,31,36, 53 , with a wide range of applications in decision-making problems, see, e.g., Ref. $5,7,23,32,34,35,41,42,45,46$.

Recently, a heavy criticism of all fuzzy AHP methods was published in Ref. 55 . However, as was shown in Ref. 14 , the criti- 
cism provided in Ref. 55 is not justified. It is based on arguments that contradict commonly accepted results of fuzzy set theory and on characteristics shared with crisp AHP and commonly accepted by the research community. Further, in Ref. 14,30, the importance of employing the constrained fuzzy arithmetic instead of the standard fuzzy arithmetic in the fuzzy extension of AHP was emphasized in order to handle the fuzziness appropriately.

In order to properly capture the vagueness of preference information in sorting problems, the FAHPSort method will be introduced in this paper by employing constrained fuzzy arithmetic (Ref. 29,31) in the fuzzy extension of AHPSort (Ref. 21 ). Triangular fuzzy numbers will be used in FAHPSort as they are most frequently used to model the meaning of linguistic terms in Saaty's scale (see the literature review in Ref. 20 ). However, let us note that FAHPSort can be easily generalized to any other type of fuzzy numbers (including trapezoidal fuzzy numbers) defined by a set of $\alpha$-cuts; the formulas in Section 3 can be used to compute the lower and upper boundary values of the $\alpha$-cuts of the fuzzy numbers.

The new FAHPSort method introduced in this paper combines strengths of the AHPSort method and fuzzy methods. In particular, similarly to AHPSort, it is suitable for sorting problems and it does not require pairwise comparisons of alternatives with respect to criteria from decision makers, which leads to reducing the number of pairwise comparisons required from the decision maker in decision problems with a large set of alternatives. Further, by utilizing fuzzy numbers, it can properly capture the vagueness on preference information provided by decision makers. On the other hand, the weakness of FAHPSort, similarly to AHPSort, is that it requires additional information from decision makers regarding the profiles representing the classes for each criterion.

The remaining of the paper is organized as follows: In Section 2, the AHPSort method is briefly described and basic notions of triangular fuzzy numbers are summarized. In Section 3, the FAHPSort method is presented, and in Section 4, the properties of the method are described and proved. The method is then illustrated with a sorting problem in Section 5, and finally, conclusion is provided in Section 6 . 


\section{Preliminaries}

In this section, basic notions of pairwise comparison matrices in AHP and triangular fuzzy numbers are recalled, and the AHPSort method is summarized briefly.

\subsection{Pairwise comparison matrices}

A (multiplicative) pairwise comparison matrix of $n$ objects $o_{1}, \ldots, o_{n}$ is a square matrix $A=\left\{a_{i j}\right\}_{i, j=1}^{n}$ whose elements $a_{i j}$ express the intensity of preference of object $o_{i}$ over object $o_{j}$. The pairwise comparison matrix is required to be reciprocal, i.e. $a_{j i}=\frac{1}{a_{i j}}, i, j=1, \ldots, n$. The pairwise comparisons in the matrix are usually done by using the Saaty scale containing integers $1-9$ and their reciprocals.

A pairwise comparison matrix $A=\left\{a_{i j}\right\}_{i, j=1}^{n}$ is said to be (multiplicatively) consistent if $a_{i j}=a_{i k} a_{k j}, i, j, k=1, \ldots, n$. Multiplicative consistency is very difficult to reach, especially for pairwise comparison matrices of large dimensions. Therefore, an acceptable level of inconsistency is usually defined. According to Saaty Ref. 44, a pairwise comparison matrix $A$ is said to be acceptably inconsistent if $C R=C I / R I<0.1$, where $C R$ is the Consistency Ratio, $C I=(\lambda-n) /(n-1)$ is the Consistency Index, $\lambda$ is the maximal eigenvalue of $A, n$ is the size of $A$, and $R I$ is the Random Index for matrices of size $n$ provided, e.g., in Ref. 50 . If pairwise comparison matrix $A$ is not acceptably inconsistent, the decision maker is asked to re-evaluate his or her pairwise comparisons, or a method for improving consistency of the pairwise comparison matrix (such as the one introduced in Ref. 27 ) can be applied.

Beside the consistency based on the multiplicative consistency, there exist various other consistency conditions, see e.g. Ref. 9,17,47 . Among these, the weak-consistency condition Ref. 47 is very natural and especially easy to fulfill for a decision maker.

Priorities $w_{1}, \ldots, w_{n}$ of objects expressing the relative preference of each object with respect to the other objects are obtained from pairwise comparison matrix $A$. The geometric mean method Ref. 11 or the maximal eigenvector method Ref. 44 are usually used for this purpose. Nevertheless, also other methods such as the weighted least squares method (Ref. 10 ), the additive normalization method (Ref. 44 ), or the cosine maximization method (Ref. 26 ) can be used for this purpose. The priorities are usually normalized so that $\sum_{i=1}^{n} w_{i}=$ 
$1, w_{i}>0, i=1, \ldots, n$, in order to get uniqueness. The normalization is done by dividing the priorities by constant $c=\sum_{i=1}^{n} w_{i}>0$.

\subsection{AHPSort}

In this subsection, the AHPSort method is briefly reviewed. For a more detailed description of the method, see Ref. 21 .

Let us assume a sorting problem with $n$ criteria $c_{1}, \ldots, c_{n}$ and $m$ alternatives $a_{1}, \ldots, a_{m}$. First, classes $C_{1}, \ldots, C_{p}$ to which the alternatives will be sorted have to be defined. These classes can be for example $C_{1}-$ satisfactory alternatives, $C_{2}-$ average alternatives, $C_{3}-$ unsatisfactory alternatives. Each class has to be characterized for each criterion. This is done by defining a limiting or a central profile on each criterion for each class. The limiting profiles $l p_{i j}, i=1, \ldots, p-1, j=$ $1, \ldots, n$, are "boundaries" between two neighboring classes $C_{i}$ and $C_{i+1}, i=1, \ldots, p-1$, for the criterion $c_{j}, j \in\{1, \ldots, n\}$. Sometimes, it is difficult to define limiting profiles, for example when the area of decision is new. In this case, central profiles are preferred. The central profiles $c p_{i j}, i=1, \ldots, p, j=1, \ldots, n$, are "typical examples" of performances belonging to classes $C_{i}, i=1, \ldots, p$, according to a given criterion $c_{j}, j=1, \ldots, n$.

For example, let us assume a decision maker who wants to sort different job offers according to the criterion $c_{1}$ - monthly salary into 3 classes $C_{1}-$ satisfactory job offers, $C_{2}-$ average job offers, $C_{3}-$ unsatisfactory job offers. The decision maker might define the limiting profiles as $l p_{11}=2500 €$ and $l p_{21}=1200 €$, which means that any salary above $2500 €$ is satisfactory, any salary between $1200 €$ and $2500 €$ is average, and any salary below $1200 €$ is unsatisfactory for the decision maker. Alternatively, the decision maker might define central profiles as e.g. $c p_{11}=3500 € c p_{21}=2000 €$ and $c p_{31}=800$ $€$, which means that a salary of $3500 €$ is the typical satisfactory salary, a salary of $2000 €$ constitutes the typical average salary, and $800 €$ is the typical unsatisfactory salary for him or her.

Once the limiting or central profiles are defined for each criterion, each alternative $a_{k}, k=1, \ldots, m$, is compared pairwisely with each profile with respect to each criterion $c_{j}, j=1, \ldots, n$. This means that, for each alternative $a_{k}$, we construct $n$ pairwise comparison matrices $M_{k j}=\left\{m_{i l}\right\}_{i, l=1}^{q+1}, j=1, \ldots, n$, where $q=p-1$ for limiting profiles and $q=p$ for central profiles. In this matrix $M_{k j}$, the alternative $a_{k}$ is compared pairwisely with $q$ profiles with respect to the criterion 
$c_{j}, j \in\{1, \ldots, n\}$.

The pairwise comparisons of the profiles for each criterion $c_{j}, j \in$ $\{1, \ldots, n\}$, are done once, and then they are reused in pairwise comparison matrices of each alternative evaluated with respect to the given criterion. Thus, for example, for $m=10$ alternatives and $q=3$ profiles, the following pairwise comparisons are required from the decision maker with respect to one criterion $c_{j}, j \in\{1, \ldots, n\}$. The 3 profiles are compared pairwisely by $\frac{3 \cdot 2}{2}=3$ pairwise comparisons, which are then utilized in the pairwise comparison matrices of each alternative evaluated with respect to criterion $c_{j}$. Further, each of the 10 alternatives is compared pairwisely with each profile, which requires $10 \cdot 3=30$ pairwise comparisons. Therefore, 33 pairwise comparisons are required from the decision maker to compare 10 alternatives with respect to one criterion. When the classic AHP is used, the decision maker has to provide more pairwise comparisons; exactly $\frac{10 \cdot 9}{2}=45$. Thus, using AHPSort method, the decision maker is spared 12 pairwise comparisons for every single criterion present in the decisionmaking problem (for, e.g., five criteria, $12 * 5=60$ pairwise comparisons are spared). With an increasing number of alternatives compared in the decision-making problem the number of spared pairwise comparisons gets significantly higher. For example, for 20 alternatives, the number of spared pairwise comparisons for every single criterion is 127 since only 63 pairwise comparisons are required in AHPSort compared to 190 pairwise comparisons required in AHP (thus, e.g., for five criteria, $5^{*} 127=635$ pairwise comparisons are spared).

To compare the objects pairwisely, the Saaty scale of integers $1-9$ with assigned linguistic terms expressing the intensities of preference of one object over another is used. In order to verify that the decision maker is not too inconsistent in his or her statements, a consistency check is performed. Saaty's consistency ratio can be used to verify acceptable inconsistency based on the multiplicative consistency property.

From each pairwise comparison matrix $M_{k j}, j \in\{1, \ldots, n\}$, of the alternative $a_{k}, k \in\{1, \ldots, m\}$, and the profiles for the given criterion $c_{j}, j \in\{1, \ldots, n\}$, the priority $w_{k j}$ of the alternative $a_{k}$ and the priorities $w p_{k i j}, i=1, \ldots, q$, of the profiles are obtained. For this purpose, the maximal eigenvalue method Ref. 44 or the geometric mean method Ref. 11 are usually used. Analogously, a pairwise comparison matrix of the criteria $c_{1}, \ldots, c_{n}$ is constructed, and the weights $v_{j}, j=1, \ldots, n$, of the criteria are derived from this matrix. 
In the next step, the weights $v_{j}$ of the criteria $c_{j}, j=1, \ldots, n$, and the priorities $w_{k j}$ of the alternative $a_{k}, k \in\{1, \ldots, m\}$, with respect to each criterion $c_{j}, j=1, \ldots, n$, are aggregated into the overall priority $o_{k}$ of the alternative $a_{k}$ by using the weighted arithmetic mean as

$$
o_{k}=\sum_{j=1}^{n} v_{j} \cdot w_{k j}, \quad k \in\{1, \ldots, m\} .
$$

Analogously, the weights $v_{j}$ of the criteria $c_{j}, j=1, \ldots, n$, and the priorities $w p_{k i j}$ of the profiles $l p_{i j}, i \in\{1, \ldots, p-1\}, j=1, \ldots, n$, or $c p_{i j}, i \in\{1, \ldots, p\}, j=1, \ldots, n$, with respect to each criterion $c_{j}, j=$ $1, \ldots, n$, and corresponding to the alternative $a_{k}, k \in\{1, \ldots, m\}$, are aggregated into the overall priorities $o p_{k i}$ of the limiting or central profiles as

$$
o p_{k i}=\sum_{j=1}^{n} v_{j} \cdot w p_{k i j}, \quad k \in\{1, \ldots, m\}, i=1, \ldots, q,
$$

where $q=p-1$ for limiting profiles and $q=p$ for central profiles.

Note that due to the weighted-arithmetic-mean aggregation formulas (1) and (2), AHPSort is a compensatory method. This means that poor performance of alternatives or profiles with respect to some of the criteria can be compensated by good performance with respect to other criteria.

Once we obtain the overall priority $o_{k}$ of the alternative $a_{k}$ and the overall priorities $o p_{k i}, i=1, \ldots, q$, of the limiting or central profiles, the alternative $a_{k}$ can be sorted into one of the classes. The assignment rules depend on the type of profiles used in the model:

When limiting profiles are used to define the classes, the alternative $a_{k}$ is sorted according to the following assignment rules:

$$
\begin{array}{ll}
a_{k} \in C_{1} & \text { when } o_{k} \geq o p_{k 1}, \\
a_{k} \in C_{i}, i=2, \ldots, p-1 & \text { when } o p_{k(i-1)}>o_{k} \geq o p_{k i}, \\
a_{k} \in C_{p} & \text { when } o p_{k(p-1)}>o_{k} .
\end{array}
$$

In the case when central profiles used to define the classes, the alternative $a_{k}$ is assigned to the class whose priority of the central profile is the nearest to the priority of $a_{k}$. That is, 


$$
\begin{array}{ll}
a_{k} \in C_{1} & \text { when } o o_{k}>o p_{k 1} \\
a_{k} \in C_{i}, i=\{1, \ldots, p-1\} & \text { when } o p_{k i} \geq o o_{k}>o p_{k(i+1)} \\
a_{k} \in C_{i+1}, i=\{1, \ldots, p-1\} & \text { and }\left(o p_{k i}-o_{k}\right)<\left(o p_{k}-o p_{k(i+1)}\right) \\
& \text { when } o p_{k i}>o_{k} \geq o p_{k(i+1)} \\
a_{k} \in C_{i}, i=\{1, \ldots, p-1\} & \text { and }\left(o p_{k i}-o_{k}\right)>\left(o o_{k}-o p_{k(i+1)}\right) \\
& \text { when } o p_{k i} \geq o_{k}>o p_{k(i+1)} \\
& \text { and }\left(o p_{k i}-o_{k}\right)=\left(o_{k}-o p_{k(i+1)}\right) \\
a_{k} \in C_{i+1}, i=\{1, \ldots, p-1\} & \text { with an optimistic vision, } \\
& \text { when } o p_{k i}>o_{k} \geq o p_{k(i+1)} \\
& \text { and }\left(o p_{k i}-o_{k}\right)=\left(o k-o p_{k(i+1)}\right) \\
a_{k} \in C_{p} & \text { with a pessimistic vision, } \\
& \text { when } o k<p_{k p} .
\end{array}
$$

According to Ref. 21 , it is also possible to derive limiting-profiles priorities $o p_{k i}^{*}, i=1, \ldots, p-1$, from the central-profiles priorities in the form $o p_{k i}^{*}=\left(o p_{k i}+o p_{k(i+1)}\right) / 2$ and then the alternatives can be sorted into the classes using the assignment rules for limiting profiles. However, let us note that different results might be obtained by this procedure.

\subsection{Triangular fuzzy numbers and fuzzy pair- wise comparison matrices}

In this subsection, necessary basic notions of triangular fuzzy numbers and fuzzy AHP used for the fuzzy extension of the AHPSort method are given.

A triangular fuzzy number $\widetilde{c}$ has a membership function given as

$$
\widetilde{c}(x)=\left\{\begin{array}{cl}
\frac{x-c^{L}}{c^{M}-c^{L}}, & c^{L}<x<c^{M}, \\
1, & x=c^{M}, \\
\frac{c^{U}-x}{c^{U}-c^{M}}, & c^{M}<x<c^{U}, \\
0, & \text { otherwise, }
\end{array}\right.
$$

where $c^{L}$ and $c^{U}$ are called the lower and upper boundary values of the triangular fuzzy number $\widetilde{c}$, and $c^{M}$ is called the middle value of the triangular fuzzy number $\widetilde{c}$. Every triangular fuzzy number can be uniquely described by a triplet of these values; the notation $\widetilde{c}=$ 
$\left(c^{L}, c^{M}, c^{U}\right)$ is used in the paper hereafter. A triangular fuzzy number $\widetilde{c}=\left(c^{L}, c^{M}, c^{U}\right)$ is said to be positive if $c^{L}>0$. The core of triangular fuzzy number $\widetilde{c}=\left(c^{L}, c^{M}, c^{U}\right)$ is the singleton set Core $(\widetilde{c})=\left\{c^{M}\right\}$, and the support is an open interval $\operatorname{Supp}(\widetilde{c})=\left(c^{L}, c^{U}\right)$.

Let $\widetilde{c}=\left(c^{L}, c^{M}, c^{U}\right)$ and $\widetilde{d}=\left(d^{L}, d^{M}, d^{U}\right)$ be two positive triangular fuzzy numbers, i.e. $c^{L}>0$ and $d^{L}>0$. In the simplified standard fuzzy arithmetic, addition, multiplication, and division of triangular fuzzy numbers are defined as $\widetilde{c}+\widetilde{d}=\left(c^{L}+d^{L}, c^{M}+d^{M}, c^{U}+d^{U}\right)$, $\widetilde{c} \cdot \widetilde{d}=\left(c^{L} d^{L}, c^{M} d^{M}, c^{U} d^{U}\right)$, and $\frac{\widetilde{c}}{\widetilde{d}}=\left(\frac{c^{L}}{d^{U}}, \frac{c^{M}}{d^{M}}, \frac{c^{U}}{d^{L}}\right)$.

As it was pointed out by Klir \& Yuan Ref. 24 , the concept of standard fuzzy arithmetic can be applied only if there are no interactions between the fuzzy numbers. In the case of any interactions between the fuzzy numbers, the concept of constrained fuzzy arithmetic should be considered instead. Let $f$ be a continuous function, $f: \mathcal{R}^{n} \rightarrow \mathcal{R}$, and let triangular fuzzy numbers $\widetilde{a}_{i}=\left(a_{i}^{L}, a_{i}^{M}, a_{i}^{U}\right), i=1, \ldots, n$, express uncertain values of an $n$-tuple of variables. Let $g\left(a_{1}, \ldots, a_{n}\right)=0$ represents a constraint imposed on the $n$-tuple of variables. Then $f\left(\widetilde{a}_{1}, \ldots, \widetilde{a}_{n}\right)$ is a triangular fuzzy number $\widetilde{c}=\left(c^{L}, c^{M}, c^{U}\right)$ whose representing values are given as follows:

$$
\begin{aligned}
c^{L} & =\min \left\{f\left(a_{1}, \ldots, a_{n}\right) ; a_{i} \in\left[a_{i}^{L}, a_{i}^{U}\right], i=1, \ldots, n, g\left(a_{1}, \ldots, a_{n}\right)=0\right\} \\
c^{M} & =f\left(a_{1}^{M}, \ldots, a_{n}^{M}\right), \\
c^{U} & =\max \left\{f\left(a_{1}, \ldots, a_{n}\right) ; a_{i} \in\left[a_{i}^{L}, a_{i}^{U}\right], i=1, \ldots, n, g\left(a_{1}, \ldots, a_{n}\right)=0\right\} .
\end{aligned}
$$

In order to defuzzify a triangular fuzzy number, the center-of-area defuzzification method (often called also the center-of-gravity method) Ref. 49 is often used because of its computational simplicity and well-accepted results. The center of area $C O A_{\widetilde{c}}$ of a triangular fuzzy number $\widetilde{c}=\left(c^{L}, c^{M}, c^{U}\right)$ is obtained by the formula

$$
C O A_{\widetilde{c}}=\frac{1}{3}\left(c^{L}+c^{M}+c^{U}\right) .
$$

For two triangular fuzzy numbers $\widetilde{c}=\left(c^{L}, c^{M}, c^{U}\right), \widetilde{d}=\left(d^{L}, d^{M}, d^{U}\right)$ and a constant $\alpha \neq 0$, the following holds:

$$
\begin{aligned}
& C O A_{\widetilde{c}+\widetilde{d}}=C O A_{\widetilde{c}}+C O A_{\widetilde{d}} \\
& C O A_{\alpha \widetilde{c}}=\alpha C O A_{\widetilde{c}} .
\end{aligned}
$$

To measure the distance of two triangular fuzzy numbers, the dissemblance index introduced in Ref. 22 is very often used because of 
its computational simplicity and well-accepted results. For two triangular fuzzy numbers $\widetilde{c}=\left(c^{L}, c^{M}, c^{U}\right)$ and $\widetilde{d}=\left(d^{L}, d^{M}, d^{U}\right)$, the dissemblance index $D(\widetilde{c}, \widetilde{d}), 0 \leq D(\widetilde{c}, \widetilde{d}) \leq 1$, is computed as

$D(\widetilde{c}, \widetilde{d})=\int_{0}^{1}\left(\left|c^{L}-d^{L}+\left(c^{M}-c^{L}+d^{L}-d^{M}\right) x\right|+\left|c^{U}-d^{U}+\left(c^{M}-c^{U}+d^{U}-d^{M}\right) x\right|\right) d x$.

$A$ (multiplicative) fuzzy pairwise comparison matrix of $n$ objects $x_{1}, \ldots, x_{n}$ is a square matrix $\widetilde{A}=\left\{\widetilde{a}_{i j}\right\}_{i, j=1}^{n}$ whose elements $\widetilde{a}_{i j}, i, j=$ $1, \ldots, n$, are triangular fuzzy numbers expressing the intensity of preference of object $x_{i}$ over object $x_{j}$. A scale of fuzzy numbers with assigned linguistic terms expressing the intensity of preference of one object over another is used for the construction of fuzzy pairwise comparison matrices. When object $x_{i}$ is more preferred than object $x_{j}$, the element $\widetilde{a}_{i j}$ of the pairwise comparison matrix $\widetilde{A}=\left\{\widetilde{a}_{i j}\right\}_{i, j=1}^{n}$ is assigned a triangular fuzzy number from the given scale expressing the intensity of preference. When object $x_{j}$ is more preferred than object $x_{i}$, the element $\widetilde{a}_{i j}$ is assigned the reciprocal of a triangular fuzzy number from the scale. Analogously as for the pairwise comparison matrix of real numbers, the fuzzy pairwise comparison matrix must be reciprocal, i.e. $\widetilde{a}_{i j}=\frac{1}{\tilde{a}_{j i}}$ for $i, j=1, \ldots, n$. On the main diagonal of a fuzzy pairwise comparison matrix, each object is compared with itself. Naturally, the objects are always equally preferred to themselves, and there is no fuzziness in these comparisons. Therefore, it is required to have $\widetilde{a}_{i i}=1, i=1, \ldots, n$, for any fuzzy pairwise comparison matrix.

The requirement of reciprocity of pairwise comparisons in a fuzzy pairwise comparison matrix is in fact an interaction on a set of fuzzy numbers. Therefore, the constrained fuzzy arithmetic is necessary for obtaining the fuzzy priorities of objects from fuzzy pairwise comparisons matrices and for aggregating the fuzzy priorities by weighted average into the overall fuzzy priorities of alternatives.

As mentioned earlier, triangular fuzzy numbers are used for the fuzzy extension of the AHPSort method in this paper. In particular, the fuzzy extension of the Saaty scale given in Tab. 1, which was defined in Ref. 33 , is applied here. Note that $\widetilde{1}=(1 / 3,1,3)$ in Tab. 1 standing for "equal preference" is equal to its reciprocal. The necessity of this special form of $\widetilde{1}$ is explained in Ref. 31 . However, we do not claim that the fuzzy extension of the Saaty scale in Tab. 1 is the only option for expressing the intensities of preference. Ideally, the membership functions of the triangular fuzzy numbers describing the 
intensities of preference should be modeled individually for every decision maker in order to reflect decision maker's subjective perception of the linguistic terms expressing the intensities of preference. The membership functions can be defined indirectly with known measures as in Ref. 20 . Alternatively, the decision makers could be supported directly in the following way. The decision analyst may ask them: "What multiple of the intensity of preference represents the best the moderate (or equal, strong, very strong, extreme) preference of one object over another object?" in order to identify the middle value of the corresponding triangular fuzzy number. And "What is the lowest / highest multiple of intensity of preference that would still represent the moderate (or equal, strong, very strong, extreme) preference of one object over another object?" in order to identify the lower and upper boundary values.

Note that instead of triangular fuzzy numbers, also trapezoidal fuzzy numbers $\widetilde{t}=\left(t^{\alpha}, t^{\beta}, t^{\gamma}, t^{\delta}\right)$ with the membership function given as

$$
\widetilde{t}(x)=\left\{\begin{array}{cl}
\frac{x-t^{\alpha}}{t^{\beta}-t^{\alpha}}, & t^{\alpha}<x<t^{\beta}, \\
1, & t^{\beta} \leq x \leq t^{\gamma}, \\
\frac{t^{\delta}-x}{t^{\delta}-t^{\gamma}}, & t^{\gamma}<x<t^{\delta}, \\
0, & \text { otherwise, }
\end{array}\right.
$$

or arbitrary fuzzy numbers described by their $\alpha$-cuts could be used to model vagueness of linguistic terms used to provide pairwise comparisons. In that case all concepts described in this section for triangular fuzzy numbers would need to be extended appropriately. For this purpose, Ref. 30 could be used as a guide.

Let us note that it would also be possible to use intervals, type- 2 fuzzy sets (Ref. 54 ), or intuitionistic fuzzy sets (Ref. 1 ) to model the linguistic terms. However, intervals do not have any degrees of membership and thus do not model uncertainty on the linguistic terms very well. On the other hand, type-2 fuzzy sets and intuitive fuzzy sets are rather complex and require more information from the decision maker.

\section{FAHPSort}

In this section, a fuzzy extension of the AHPSort method into the FAHPSort method will be proposed. As mentioned in the previous 
Table 1: Fuzzified Saaty scale.

\begin{tabular}{ccl}
\hline $\begin{array}{c}\text { Fuzzy } \\
\text { number }\end{array}$ & $\begin{array}{c}\text { Membership } \\
\text { function }\end{array}$ & Linguistic term \\
\hline 1 & $(1 / 3,1,3)$ & equal preference \\
3 & $(1,3,5)$ & moderate preference \\
5 & $(3,5,7)$ & strong preference \\
7 & $(5,7,9)$ & very strong preference \\
9 & $(7,9,9)$ & extreme preference \\
\hline
\end{tabular}

sections, triangular fuzzy numbers will be used here for the fuzzy extension. However, the method can be easily generalized to any type of fuzzy numbers.

Same as in the AHPSort method, in the FAHPSort method too, we assume a sorting problem with $n$ criteria $c_{1}, \ldots, c_{n}, m$ alternatives $a_{1}, \ldots, a_{m}$, and $p$ classes $C_{1}, \ldots, C_{p}$ to which the alternatives will be sorted. The classes and the limiting or central profiles for each criterion are defined in the same way as in the AHPSort method. Note that no fuzziness is considered in this phase; the decision maker provides the profiles as particular values of the criteria in numerical or linguistic form exactly in the same way as in the AHPSort method. The fuzziness is employed in the later phase when the alternatives are compared pairwisely with these profiles using linguistic terms expressing the intensity of preference of one compared object over another.

Note that if we considered fuzziness at the stage of defining limiting or central profiles for the criteria, the profiles would be represented by fuzzy numbers instead of numerical values or linguistic terms. However, this generalization would have no impact on the method; the whole procedure would remain exactly the same. The decision makers would only have to compare the alternatives with the profiles that are represented by fuzzy numbers. However, this comparison would be still done in the same way, by using linguistic terms from the Saaty scale.

Once the limiting or central profiles are defined, each alternative $a_{k}, k=1, \ldots, m$, is compared pairwisely with each profile with respect to each criterion $c_{j}, j=1, \ldots, n$. Unlike in the original AHPSort method, in the FAHPSort method, the intensities of preferences 
in the Saaty scale are described by triangular fuzzy numbers in order to capture the vagueness in meaning of linguistic terms. In this paper, we use the fuzzified Saaty scale given in Tab. 1. It means that, for each alternative $a_{k}$, we construct $n$ fuzzy pairwise comparison matrices $\widetilde{M}_{k j}=\left\{\widetilde{m}_{i k}\right\}_{i, k=1}^{q+1}, j=1, \ldots, n$, of triangular fuzzy numbers. In fuzzy matrix $\widetilde{M}_{k j}$, the alternative $a_{k}$ is compared pairwisely with $q$ profiles with respect to the criterion $c_{j}, j \in\{1, \ldots, n\}$, where $q=p-1$ for a limiting profile and $q=p$ for a central profile.

\subsection{Verifying acceptable consistency}

In order to verify the acceptable consistency of fuzzy pairwise comparison matrices, fuzzy consistency ratio $\overline{C R}$ defined in Ref. 29 is computed. For a fuzzy pairwise comparison matrix $\widetilde{M}=\left\{m_{i j}\right\}_{i, j=1}^{n}$, fuzzy consistency ratio $\widetilde{C R}$ is obtained as

$$
\widetilde{C R}=\frac{\widetilde{C I}}{R I}=\frac{\widetilde{\lambda}-n}{R I(n-1)}=\left(\frac{\lambda^{L}-n}{R I(n-1)}, \frac{\lambda^{M}-n}{R I(n-1)}, \frac{\lambda^{U}-n}{R I(n-1)}\right)
$$

where $\widetilde{\lambda}=\left(\lambda^{L}, \lambda^{M}, \lambda^{U}\right)$ is the fuzzy maximal eigenvalue of the fuzzy pairwise comparison matrix $\widetilde{M}$ obtained by the formulas

$$
\begin{aligned}
\lambda^{L}=\min \left\{\max \left\{\lambda ;|M-\lambda I|=0, M=\left\{m_{i j}\right\}_{i, j=1}^{n}\right\} ;\right. \\
\left.m_{i j} \in\left[a_{i j}^{L}, m_{i j}^{U}\right], m_{i j}=\frac{1}{m_{j i}}, m_{i i}=1, i, j=1, \ldots, n\right\},
\end{aligned}
$$

$$
\begin{gathered}
\lambda^{M}=\quad \max \left\{\lambda ;\left|M^{M}-\lambda I\right|=0, M^{M}=\left\{m_{i j}^{M}\right\}_{i, j=1}^{n}\right\} \\
\lambda^{U}=\max \left\{\max \left\{\lambda ;|M-\lambda I|=0, M=\left\{m_{i j}\right\}_{i, j=1}^{n}\right\} ;\right. \\
\left.m_{i j} \in\left[m_{i j}^{L}, m_{i j}^{U}\right], m_{i j}=\frac{1}{m_{j i}}, m_{i i}=1, i, j=1, \ldots, n\right\},
\end{gathered}
$$

proposed in Ref. 29 . The formulas (11) and (13) are based on the constrained fuzzy arithmetic. The interaction among the fuzzy 
numbers is the reciprocity property of pairwise comparisons which is a key property of AHP Ref. 43 . Thus, the $m_{i j}, i, j=1, \ldots, n$, that minimize $\lambda^{L}$, maximize $\lambda^{U}$, and preserve the reciprocity of pairwise comparisons are searched for.

According to Ref. 29 , a fuzzy pairwise comparison matrix $\widetilde{M}$ is said to be acceptably inconsistent if

$$
C O A_{\widetilde{C R}}<0.1
$$

where $C O A_{\widetilde{C R}}$ is the center of area of $\widetilde{C R}$ obtained by (6).

\subsection{Deriving fuzzy priorities}

We are at the stage when we have for the given criterion $c_{j}, j \in$ $\{1, \ldots, n\}$, a fuzzy pairwise comparison matrix $\widetilde{M}_{k j}, j \in\{1, \ldots, n\}$, of the alternative $a_{k}, k \in\{1, \ldots, m\}$, and of the profiles. The fuzzy priority $\widetilde{w}_{k j}$ of the alternative $a_{k}$ and the fuzzy priorities $\widetilde{w p}_{k i j}, i=$ $1, \ldots, q$, of the profiles with respect to the criterion $c_{j}$ have to be derived from this fuzzy pairwise comparison matrix. An extension of the geometric-mean method (Ref. 11 ) is applied for this purpose.

The fuzzy geometric mean method proposed in Ref. 31 is adopted in our paper since it is based on the constraint fuzzy arithmetic and preserves the reciprocity of pairwise comparisons. According to Ref. 31 , fuzzy priorities $\widetilde{w}_{i}=\left(w_{i}^{l}, w_{i}^{M}, w_{i}^{U}\right), i=1, \ldots, n$, of objects (in our case alternatives and limiting or central profiles) are obtained from a fuzzy pairwise comparison matrix $\widetilde{M}=\left\{\widetilde{m}_{i j}\right\}_{i, j=1}^{n}, \widetilde{m}_{i j}=$ $\left(m_{i j}^{L}, m_{i j}^{M}, m_{i j}^{U}\right)$, in the form

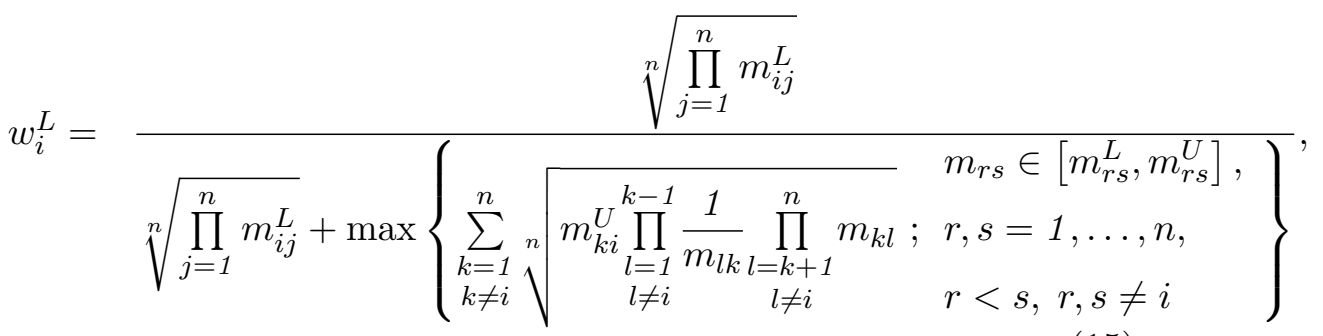

$$
\begin{aligned}
& w_{i}^{M}=\frac{\sqrt[n]{\prod_{j=1}^{n} m_{i j}^{M}}}{\sum_{k=1}^{n} \sqrt[n]{\prod_{j=1}^{n} m_{k j}^{M}}}
\end{aligned}
$$




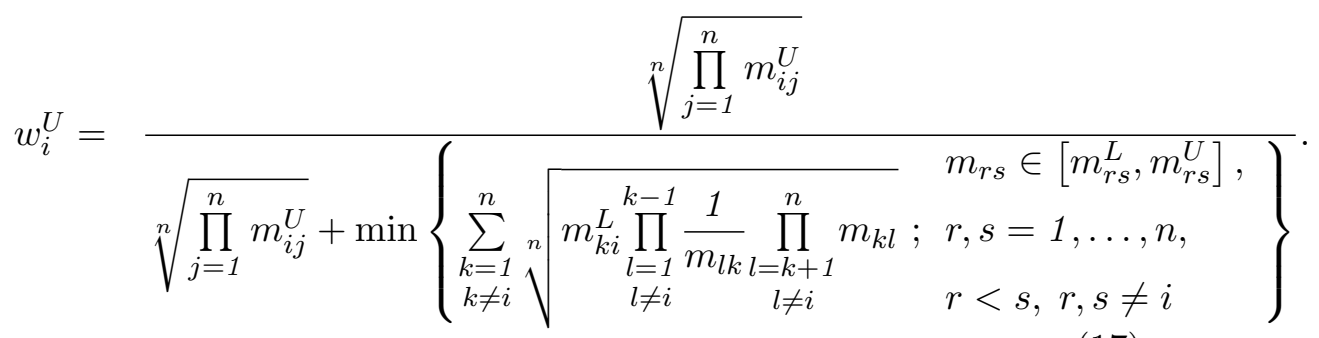

Note that it is also possible to derive crisp priorities $w_{i}, i=1, \ldots, n$, from fuzzy pairwise comparison matrices instead of fuzzy priorities $\widetilde{w}_{i}, i=1, \ldots, n$, by applying one of the existing methods (e.g. Ref. 40 or Ref. 15 ). However, such an approach, in our opinion, does not reflect properly the vagueness of preference information provided by decision makers in fuzzy pairwise comparison matrices. That is why we prefer to use fuzzy priorities in the FAHPSort method.

\subsection{Aggregation of the fuzzy priorities}

Once all fuzzy priorities $\widetilde{w}_{k j}$ of alternative $a_{k}, k \in\{1, \ldots, m\}$, with respect to each criterion $c_{j}, j=1, \ldots, n$, are derived, they are aggregated into the overall fuzzy priority $\widetilde{o}_{k}=\left(o_{k}^{L}, o_{k}^{M}, o_{k}^{U}\right)$ of alternative $a_{k}$ by the fuzzy weighted average proposed in Ref. 31 :

$$
\begin{gathered}
o_{k}^{L}=\min \left\{\frac{\sum_{i=1}^{n} \sqrt[n]{\prod_{j=1}^{n} m_{i j}} \cdot w_{k i}^{L}}{\sum_{i=1}^{n} \sqrt[n]{\prod_{j=1}^{n} m_{i j}}} ; m_{r s} \in\left[m_{r s}^{L}, m_{r s}^{U}\right], m_{s r}=\frac{1}{m_{r s}}\right. \\
\left.r, s=1, \ldots, n, r<s, m_{r r}=1, r=1, \ldots, n\right\} \\
o_{k}^{M}=\frac{\sum_{i=1}^{n} \sqrt[n]{\prod_{j=1}^{n} m_{i j}^{M}} \cdot w_{k i}^{M}}{\sum_{i=1}^{n} \sqrt[n]{\prod_{j=1}^{n} m_{i j}^{M}}}
\end{gathered}
$$




$$
\begin{gathered}
o_{k}^{U}=\max \left\{\frac{\sum_{i=1}^{n} \sqrt[n]{\prod_{j=1}^{n} m_{i j}} \cdot w_{k i}^{U}}{\sum_{i=1}^{n} \sqrt[n]{\prod_{j=1}^{n} m_{i j}}} ; m_{r s} \in\left[m_{r s}^{L}, m_{r s}^{U}\right], m_{s r}=\frac{1}{m_{r s}}\right. \\
\left.r, s=1, \ldots, n, r<s, m_{r r}=1, r=1, \ldots, n\right\}
\end{gathered}
$$

The fuzzy weighted average (18)-(20) is based on the constrained fuzzy arithmetic and, so far as we are aware, it is the only fuzzy extension of the weighted average preserving the reciprocity of the pairwise comparisons. Note that the fuzzy pairwise comparison matrix of criteria $\widetilde{M}=\left\{\widetilde{m}_{i j}\right\}_{i, j=1}^{n}, \widetilde{m}_{i j}=\left(m_{i j}^{L}, m_{i j}^{M}, m_{i j}^{U}\right)$, is employed in the formulas (18)-(20) instead of the fuzzy weights of the criteria to calculate the overall fuzzy priorities of $a_{k}$. This step is crucial in order to preserve the reciprocity property of pairwise comparisons of the criteria.

Analogously, the overall fuzzy priority $\widetilde{o p}_{k i}$ of each limiting or central profile is obtained; fuzzy priorities $\widetilde{w}_{k i}=\left(w_{k i}^{L}, w_{k i}^{M}, w_{k i}^{U}\right), i=$ $1, \ldots, n$, of alternative $a_{k}$ in the formulas (18)-(20) are just replaced by corresponding fuzzy priorities $\widetilde{w p}_{k i j}=\left(w p_{k i j}^{L}, w p_{k i j}^{M}, w p_{k i j}^{U}\right), i=$ $1, \ldots, n$, of the given profile.

\subsection{Sorting of the alternatives}

Once we obtain the overall fuzzy priority $\widetilde{o}_{k}$ of alternative $a_{k}, k \in$ $\{1, \ldots, m\}$, and the corresponding overall fuzzy priorities $\widetilde{o p}_{k l}, l=$ $1, \ldots, q$, of the limiting or central profiles, the alternative $a_{k}$ can be sorted into one of the classes. The assignment rules depend on the type of profiles used in the model. We propose a fuzzy extension of the assignment rules (3) and (4) for triangular fuzzy numbers as described in the following subsections.

\subsubsection{Assignment rules for the limiting profiles}

When limiting profiles are used to define the classes, the center of area $C O A$ of the overall fuzzy priority $\widetilde{o}_{k}$ of the alternative $a_{k}$ and the center of area of the corresponding overall fuzzy priorities $\widetilde{o p}_{k l}$ of the limiting profiles are computed using the formula (6). They are then 
compared as in the original AHPSort method. This means that:

$$
\begin{array}{ll}
a_{k} \in C_{1} & \text { when } C O A_{\widetilde{o}_{k}} \geq C O A_{\widetilde{o p}_{k 1}} \\
a_{k} \in C_{i}, i=2, \ldots, p-1 & \text { when } C O A_{\widetilde{o p}_{k(i-1)}}>C O A_{\widetilde{o}_{k}} \geq C O A_{\widetilde{o p}_{k i}}, \\
a_{k} \in C_{p} & \text { when } C O A_{\widetilde{o p}_{k(p-1)}}>C O A_{\widetilde{o}_{k}} .
\end{array}
$$

\subsubsection{Assignment rules for the central profiles}

In case of central profiles used to define the classes, we propose the following two extensions of the assignment rule (4).

(a) One possibility is to defuzzify the overall fuzzy priorities by the center-of-area method (6) and then to compare the obtained crisp values analogously as in (4):

$$
\begin{aligned}
& a_{k} \in C_{1} \quad \text { when } C O A_{\widetilde{o}_{k}}>C O A_{\widetilde{o p}_{k 1}} \text {, } \\
& a_{k} \in C_{i}, i=\{1, \ldots, p-1\} \quad \text { when } C O A_{\widetilde{o p}_{k i}} \geq C O A_{\widetilde{o}_{k}}>C O A_{\widetilde{o p}}{ }_{k(i+1)} \\
& \text { and }\left(C O A_{\widetilde{o p}_{k i}}-C O A_{\widetilde{o}_{k}}\right)<\left(C O A_{\widetilde{\sigma}_{k}}-C O A_{\widetilde{o p}_{k(i+1)}}\right) \text {, } \\
& a_{k} \in C_{i+1}, i=\{1, \ldots, p-1\} \quad \text { when } C O A_{\widetilde{o p}_{k i}}>C O A_{\widetilde{\sigma}_{k}} \geq C O A_{\widetilde{o p}_{k(i+1)}} \\
& \text { and }\left(C O A_{\widetilde{o p}_{k i}}-C O A_{\widetilde{\sigma}_{k}}\right)>\left(C O A_{\widetilde{\sigma}_{k}}-C O A_{\widetilde{o p}_{k(i+1)}}\right) \text {, } \\
& a_{k} \in C_{p} \quad \text { when } C O A_{\widetilde{o}_{k}}<C O A_{\widetilde{o p}_{k p}} \text {, } \\
& a_{k} \in C_{i}, i=\{1, \ldots, p-1\} \quad \text { when } C O A_{\widetilde{o p}} \geq C O A_{\widetilde{o}_{k}}>C O A_{\widetilde{o p}}{ }_{k(i+1)} \\
& \text { and }\left(C O A_{\widetilde{o p}_{k i}}-C O A_{\widetilde{\sigma}_{k}}\right)=\left(C O A_{\widetilde{o}_{k}}-C O A_{\widetilde{o p}_{k(i+1)}}\right) \\
& \text { with an optimistic vision, } \\
& a_{k} \in C_{i+1}, i=\{1, \ldots, p-1\} \quad \text { when } C O A_{\widetilde{o p}}>C O A_{\widetilde{o}_{k}} \geq C O A_{\widetilde{o p}}{ }_{k(i+1)} \\
& \text { and }\left(C O A_{\widetilde{o p}_{k i}}-C O A_{\widetilde{o}_{k}}\right)=\left(C O A_{\widetilde{\sigma}_{k}}-C O A_{\widetilde{o p}_{k(i+1)}}\right) \text {, } \\
& \text { with a pessimistic vision. }
\end{aligned}
$$

(b) An alternative way to sort the alternatives is to measure the distance $D\left(\widetilde{o}_{k}, \widetilde{o p}_{k l}\right)$ of the fuzzy priority $\widetilde{o}_{k}$ of the alternative $a_{k}, k \in\{1, \ldots, m\}$, from the fuzzy priorities $\widetilde{o p}_{k l}, l=1, \ldots, p$, of the central profiles directly without defuzzifying them first. The alternative is sorted to the class whose fuzzy priority of the central profile is the nearest to the fuzzy priority of the alternative. To measure the distance between the fuzzy priorities, 
the dissemblance index (8) is applied. The alternatives are then sorted according to these rules:

$$
\begin{array}{ll}
a_{k} \in C_{1} & \text { when } D\left(\widetilde{o p}_{k 1}, \widetilde{o}_{k}\right)=\min _{j=1, \ldots, p} D\left(\widetilde{o p}_{k j}, \widetilde{o}_{k}\right) \\
& \text { and } D\left(\widetilde{o p}_{k 1}, \widetilde{o}_{k}\right)<D\left(\widetilde{o p}_{k 2}, \widetilde{o}_{k}\right), \\
a_{k} \in C_{i}, i \in\{2, \ldots, p-1\} \quad & \text { when } D\left(\widetilde{o p}_{k i}, \widetilde{o}_{k}\right)=\min _{j=1, \ldots, p} D\left(\widetilde{o p}_{k j}, \widetilde{o}_{k}\right) \\
& \text { and } D\left(\widetilde{o p}_{k(i-1)}, \widetilde{o}_{k}\right)>D\left(\widetilde{o p}_{k i}, \widetilde{o}_{k}\right)<D\left(\widetilde{o p}_{k(i+1)}, \widetilde{o}_{k}\right), \\
& \text { when } D\left(\widetilde{o p}_{k p}, \widetilde{o}_{k}\right)=\min _{j=1, \ldots, p} D\left(\widetilde{o p}_{k j}, \widetilde{o}_{k}\right) \\
a_{k} \in C_{p} & \text { and } D\left(\widetilde{o p}_{k p}, \widetilde{o}_{k}\right)<D\left(\widetilde{o p}_{k(p-1)}, \widetilde{o}_{k}\right), \\
a_{k} \in C_{i}, i \in\{1, \ldots, p-1\} & \text { when } D\left(\widetilde{o p}_{k i}, \widetilde{o}_{k}\right)=\min _{j=1, \ldots, p} D\left(\widetilde{o p} k j, \widetilde{o}_{k}\right) \\
& \text { and } D\left(\widetilde{o p}_{k i}, \widetilde{o}_{k}\right)=D\left(\widetilde{o p} k(i+1), \widetilde{o}_{k}\right) \\
& \text { with an optimistic vision, } \\
a_{k} \in C_{i+1}, i \in\{1, \ldots, p-1\} & \text { when } D\left(\widetilde{o p}_{k(i+1)}, \widetilde{o}_{k}\right)=\min _{j=1, \ldots, p} D\left(\widetilde{o p}_{k j}, \widetilde{o}_{k}\right) \\
& \text { and } D\left(\widetilde{o p}_{k(i+1)}, \widetilde{o}_{k}\right)=D\left(\widetilde{o p}_{k i}, \widetilde{o}_{k}\right) \\
& \text { with a pessimistic vision. }
\end{array}
$$

Unlike the assignment rules (22), the assignment rules (23) do not require any defuzzification. This means that the vagueness of the overall fuzzy priorities is preserved during the sorting process, and thus, no information is lost. Therefore, the assignment rules (23) could be considered to be more appropriate than the assignment rules (22) regarding the vague nature of the sorting decision-making problem.

Note that the assignment rules (22) and (23) can lead to a different sorting as they are based on different approaches to measuring distance of triangular fuzzy numbers. Nevertheless, an alternative is always sorted to the same or to two neighboring classes by the assignment rules (22) and (23). For example, it can never happen that an alternative would be sorted to class $C_{1}$ by one sorting rule and to class $C_{3}$ by the other sorting rule. The differences in sorting with the assignment rules (22) and (23) may serve for sensitivity analysis. 


\section{Properties of the FAHPSort method}

In this section, the main properties of the FAHPSort method and their demonstrations are presented.

- Independence: Sorting of an alternative $a_{i}$ does not depend on the sorting of any other alternative $a_{j}, j=1, \ldots, m, j \neq i$.

Proof 1 Profiles (both central and limiting) are defined independently of the alternatives. Each alternative $a_{i}, i \in\{1, \ldots, m\}$, is compared with the profiles independently of the other alternatives $a_{j}, j=1, \ldots, m, j \neq i$. Therefore, the sorting of the alternative $a_{i}$ is also independent of sorting of any other alternative $a_{j}, j=1, \ldots, m, j \neq i$.

- Homogeneity: If two alternatives $a_{i}$ and $a_{j}, i \neq j$, are compared identically with the profiles (either central or limiting) on all criteria, they are sorted into the same class.

Proof 2 When two alternatives are compared identically with the profiles according to each criterion, it means that their corresponding fuzzy pairwise comparison matrices are identical. Therefore, the fuzzy priorities of the alternatives obtained from these fuzzy pairwise comparison matrices are also identical, and the corresponding fuzzy priorities of the profiles are identical too. This means that the alternatives are then sorted into the same class.

- Uniqueness: Every alternative is always assigned to one class.

Proof 3 From the definition of FAHPSort, two profiles cannot be identical as they represent different classes, i.e. not even their fuzzy priorities can be identical. Therefore, according to the assignment rules for both central and limiting profiles, every alternative is sorted uniquely into one class.

- Monotonicity: If an alternative $a_{i}$ dominates an alternative $a_{j}$, it is sorted into the same or a better class.

Proof 4 Let $a_{j}$ be sorted into class $C_{\alpha}, \alpha \in\{1, \ldots, p-1\}$, and, without any loss of generality, let $a_{i}$ dominate $a_{j}$ with respect to criterion $c_{\beta}, \beta \in\{1, \ldots, n\}$. Then, the intensities of preference of $a_{i}$ over the profiles are stronger or equal compared to 
the corresponding intensities of preference of $a_{j}$ over the profiles with respect to criterion $c_{\beta}$. It means that, using the fuzzified scale from Tab. 1, the fuzzy numbers representing the intensities of preference of $a_{i}$ over the profiles are greater or equal to the fuzzy numbers representing the intensities of preference of $a_{j}$ over the profiles. Therefore, the fuzzy priority of $a_{i}$ obtained from the fuzzy pairwise comparison matrix by formulas (15)-(17) is greater or equal to the fuzzy priority of $a_{j}$ obtained from the corresponding fuzzy pairwise comparison matrix, i.e. $\widetilde{w}_{i \beta} \geq \widetilde{w}_{j \beta}$. Analogously, the fuzzy priorities of the profiles corresponding to $a_{i}$ are smaller or equal to the fuzzy priorities of the profiles corresponding to $a_{j}$, i.e. $\widetilde{w p}_{i l \beta} \leq \widetilde{w p}_{j l \beta}, l=1, \ldots, q$.

By aggregating the fuzzy priorities of alternatives and corresponding fuzzy priorities of profiles into the overall fuzzy priorities by formulas (18)-(20), the overall fuzzy priority of $a_{i}$ is greater or equal to the overall fuzzy priority of $a_{j}, \widetilde{o}_{i} \geq \widetilde{o}_{j}$, and the overall fuzzy priorities of the profiles corresponding to $a_{i}$ are smaller or equal to the overall fuzzy priorities of the profiles corresponding to $a_{j}, \widetilde{o p}_{i l} \leq \widetilde{o p}_{j l}, l=1, \ldots, q$.

Further, we need to distinguish between limiting and central profiles used in the models. In case of central profiles used to represent the classes, dissemblance indexes for the overall fuzzy priority of the given alternative and the corresponding overall fuzzy priorities of the central profiles are computed in order to sort the alternative into one of the classes. Because $a_{j}$ is sorted into the class $C_{\alpha}$, clearly the inequality $D\left(\widetilde{o}_{i}, \widetilde{o p}_{k(\alpha+1)}\right) \geq D\left(\widetilde{o}_{j}, \widetilde{o p}_{k(\alpha+1)}\right)$ holds, which means that $a_{i}$ cannot be sorted into the class $C_{\alpha+1}$ or a lower one. By consequence, $a_{i}$ has to be sorted into the class $C_{\alpha}$ or a higher one.

In case of limiting profiles used to represent the classes, the center of area is computed for the overall fuzzy priorities of the alternatives and the limiting profiles. Since $C O A_{\widetilde{o}_{j}} \geq C O A_{\widetilde{o p}_{k \alpha}}$, then $C O A_{\widetilde{o}_{i}} \geq C O A_{\widetilde{o p}_{k \alpha}}$ also holds, which means that $a_{i}$ cannot be sorted into the class $C_{\alpha+1}$ or a lower one. Therefore, $a_{i}$ has to be sorted into the class $C_{\alpha}$ or a higher one.

- Conformity: Every profile is unequivocally assigned to the class represented by that profile.

Proof 5 We need to prove that an alternative identical to the 
profile of any of the classes $C_{1}, \ldots, C_{p}$ is sorted to that class.

Let us assume alternative $a_{i}$ to be identical with the profile (either limiting or central) of class $C_{\alpha}, \alpha \in\{1, \ldots, p\}$. Then, $a_{i}$ is equally preferred to the profile of $C_{\alpha}$ according to each criterion $c_{j}, j=1, \ldots, n$. Furthermore, the intensities of preference of $a_{i}$ over the profiles of the remaining classes $C_{\beta}, \beta=1, \ldots, p, \beta \neq \alpha$, are identical with the corresponding intensities of preference of the profile of $C_{\alpha}$ over those profiles.

Using the fuzzy scale from Tab. 1, with $\widetilde{1}$ such that $\widetilde{1}=\frac{1}{\widetilde{1}}$, the fuzzy pairwise comparison matrices of $a_{i}$ and the profiles with respect to each criterion $c_{j}, j=1, \ldots, n$, are always in such a form that the rows corresponding to $a_{i}$ and to the profile of $C_{\alpha}$ are identical. Then, by using the formulas (15)-(17), it is guaranteed that $\widetilde{w p}_{i \alpha j}=\widetilde{w}_{i j}$ for each criterion $c_{j}, j=1, \ldots, n$. Therefore, by aggregating the fuzzy priorities into the overall fuzzy priorities by formulas (18)-(20), we obtain $\widetilde{o}_{i}=\widetilde{\text { op }_{i \alpha}}$. Finally, we need to distinguish between limiting and central profiles used in the model.

In case of central profiles used to define the classes, we obtain $C O A_{\widetilde{o}_{i}}=C O A_{\widetilde{o p}_{i \alpha}}$. Therefore, $C O A_{\widetilde{o p}_{i(\alpha-1)}}>C O A_{\widetilde{o}_{i}} \geq C O A_{\widetilde{o p}_{i \alpha}}$ for $\alpha=2, \ldots, p$ and $C O A_{\widetilde{o}_{i}} \geq C O A_{\widetilde{o p}_{i \alpha}}$ for $\alpha=1$, and consequently, $a_{i}$ (which is actually the profile of $C_{\alpha}$ ) is sorted into class $C_{\alpha}$, i.e. $a_{i} \in C_{\alpha}$.

In case of limiting profiles used to define the classes, we obtain $D\left(\widetilde{o}_{i}, \widetilde{o p}_{i \alpha}\right)=0$, and since $D\left(\widetilde{o}_{i}, \widetilde{o p}_{i \beta}\right)>0$ for $\beta=1, \ldots, p, \beta \neq$ $\alpha$, then $a_{i} \in C_{\alpha}$.

\section{Case study}

In order to illustrate the FAHPSort method, we applied it on a real tourism problem to advice a customer. The customer was an Italian student who wanted to be recommended a holiday package for next summer. However, he was still undecided with whom and where to travel. Therefore, three ordered sorting classes were suggested to him:

$\mathbf{C}_{1}=$ Including the holiday packages which are ideal regarding the customer's preferences.

$\mathbf{C}_{2}=$ Including the holiday packages that are not his preferred but he would buy them if he traveled with other persons and it was the 
favourite choice for them.

$\mathbf{C}_{3}=$ Including the holiday packages that he would not consider even if they were the favourite packages for other accompanying persons.

The decision process, excluding the problem structuring exercise, lasted half an hour.

\subsection{Alternatives definition}

The customer indicated that he wanted to consider only abroad European destinations (i.e. not in Italy). The tourist adviser proposed eight holiday packages including accommodation, flight and other possible transport but not meals. The departure is Rome airport for all the packages. The details of the packages are given in A.

\subsection{Criteria definition}

As the customer did not have any experience with structuring decisionmaking problems, especially for holidays, he was helped in selecting criteria. Before the meeting, we conducted a literature review on the relevant criteria considered when choosing a holiday package Ref. 2,52

. In collaboration with the customer, we constructed the following list of relevant criteria:

$\mathbf{c}_{1}=$ Price: price of the holiday package including flights, accommodation and other possible transfer fees

$\mathbf{c}_{2}=$ Quality of the accommodation: this is mainly given by the type of the accommodation such as a tent, a caravan, a hostel or a hotel specifying the number of stars

$\mathbf{c}_{3}=$ Location: the destination of the holiday including the local weather and safety

$\mathbf{c}_{4}=$ Length: the number of days of the holiday

$\mathbf{c}_{5}=$ Type of holiday: the main activity of the holiday such as sightseeing, relaxing in the seaside, visiting natural environment, etc.

The hierarchy of the decision problem is represented in Fig. 1. 


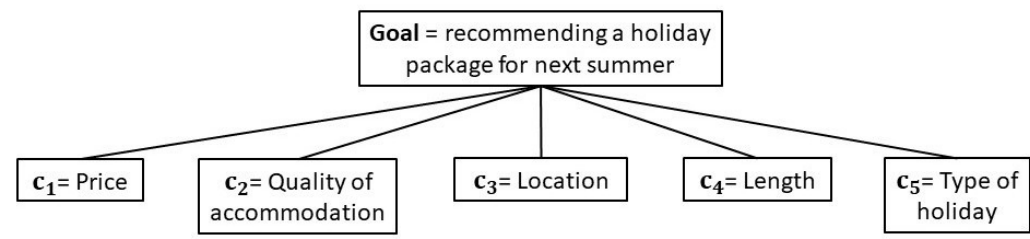

\begin{tabular}{|c|c|c|c|c|c|c|c|}
\hline $\begin{array}{c}\mathbf{a}_{1}= \\
\text { Prague }\end{array}$ & $\begin{array}{l}\mathbf{a}_{2}= \\
\text { Paris }\end{array}$ & $\begin{array}{c}\mathbf{a}_{3}= \\
\text { Amsterdam }\end{array}$ & $\begin{array}{c}\mathbf{a}_{\mathbf{4}}= \\
\text { Croatia }\end{array}$ & $\begin{array}{c}\mathbf{a}_{5}= \\
\text { Bulgaria }\end{array}$ & $\begin{array}{c}\mathbf{a}_{6}= \\
\text { Mallorca }\end{array}$ & $\begin{array}{c}\mathbf{a}_{7}= \\
\text { Norway }\end{array}$ & $\begin{array}{c}\mathbf{a}_{\mathbf{8}}= \\
\text { Switzerland }\end{array}$ \\
\hline
\end{tabular}

Figure 1: Hierarchy of the decision problem.

\subsection{Classes definition}

The three classes suggested above need to be properly defined by providing limiting or central profiles. In this case, the customer was unable to provide limiting profiles as he was not enough experienced in this kind of decision problems. Therefore, he preferred to provide typical examples (i.e. central profiles) for each class. Thus, for example, the customer defined the typical ideal holiday package representing class $C_{1}$ as a 1.5 weeks long holiday in Spain including both extreme sports and relaxing, with accommodation in a hostel and the total cost of $250 €$. The central profiles for all three classes provided by the customer are specified in Tab. 2.

\begin{tabular}{|c|c|c|c|c|c|}
\hline$c p_{i j}$ & $c_{1}$ & $c_{2}$ & $c_{3}$ & $c_{4}$ & $c_{5}$ \\
\hline$C_{1}$ & $250 €$ & hostel & Spain & 1.5 weeks & relaxing, extreme sports \\
\hline$C_{2}$ & $450 €$ & hotel ** & Croatia & 2 weeks & museums \\
\hline$C_{3}$ & $750 €$ & hotel $* * * * *$ & Germany & 1 month & wild nature \\
\hline
\end{tabular}

Table 2: Central profiles of the classes for the criteria.

\subsection{Results}

The data were collected in a structured interview, i.e. with an intervieweradministrated questionnaire. The first author of the paper read out each question and then recorded the pairwise comparison on a stan- 
dardized schedule. The collected data were then analyzed with FAHPSort (Section 3) in a software programmed in Matlab.

We present here the details of the results for the Prague package. The fuzzy pairwise comparison matrix of the criteria is given in Tab. 3 . In order to verify whether the customer was consistent in his decisions the center of area of the fuzzy consistency ratio (10) was computed and the condition (14) was verified. In particular, the fuzzy maximal eigenvalue of the fuzzy pairwise comparison matrix in Tab. 3 was obtained first by the formulas (11)-(13) as $\widetilde{\lambda}=(5,5.2375,5.8063)$. Afterwards, the fuzzy consistency ratio was computed according to the formula (10) with value $R I=1.11$ (Ref. 50 ) as

$$
\widetilde{C R}=\left(\frac{5-5}{1.11(5-1)}, \frac{5.2375-5}{1.11(5-1)}, \frac{5.8063-5}{1.11(5-1)}\right)=(0,0.0535,0.1816) \text {. }
$$

The center of area of the fuzzy consistency ratio obtained afterwards by the formula (6) is given in Tab. 3. Since this value satisfies the inequality (14), the fuzzy pairwise comparison matrix is judged as acceptably inconsistent.

Let us note that there is no need to compute the fuzzy priorities of the criteria as in the aggregation procedure the original fuzzy pairwise comparison matrix of the criteria is used instead of their fuzzy priorities in order to preserve the reciprocity of pairwise comparisons (see aggregation formulas (18)-(20)).

The Prague package has been compared with the typical examples of each class with respect to each criterion, i.e. with the central profiles given in Tab. 2. The fuzzy pairwise comparisons, the derived fuzzy priorities, and the center of area of the related fuzzy consistency ratio are given in Tab. 4-8. All fuzzy pairwise comparison matrices are consistent according to (14). This means that even though the customer provided vague preference information by using linguistic terms naturally modeled by fuzzy numbers, he was able to keep consistency.

The vague preference information provided by the customer in the fuzzy pairwise comparison matrices shown in Tab. 4-8 was then reflected properly in the fuzzy priorities of the central profiles and of the Prague package shown in the same tables. It can be noticed from the tables that the fuzzy priorities of some neighboring central profiles are even significantly overlapping for some criteria. This overlapping is a natural result of vague information provided by the customer and provides more insight in the problem. 
Let us, for example, have a closer analysis of the fuzzy priorities $(0.2706,0.4576,0.6253)$ and $(0.2204,0.3951,0.5853)$ of the profiles $c p_{14}$ and $c p_{24}$ respectively, for criterion "Length" in Tab. 7. Their significant overlapping is a natural result of the following particular situation. The customer evaluated $c p_{14}$ and $c p_{24}$ as "equally preferred". At the same time, he evaluated the pairwise comparisons of $c p_{14}$ and $c p_{24}$ with respect to $c p_{34}$ by two different intensities of preference; in particular by "extreme preference" and "strong preference", respectively. Note that if AHPSort was used in this situation instead of FAHPSort, the linguistic terms used for pairwise comparisons would have been modeled by crisp numbers, which would result in no-overlapping crisp priorities of the central profiles. This means that the crisp priorities would not properly reflect the original preference information from the customer according to which $c p_{14}$ and $c p_{24}$ are "equally preferred".

\begin{tabular}{ccccccc}
\hline & $c_{1}$ & $c_{2}$ & $c_{3}$ & $c_{4}$ & $c_{5}$ & $C O A_{\widetilde{C R}}$ \\
\hline$c_{1}$ & 1 & $(1,3,5)$ & $\left(\frac{1}{9}, \frac{1}{7}, \frac{1}{5}\right)$ & $\left(\frac{1}{5}, \frac{1}{3}, 1\right)$ & $\left(\frac{1}{7}, \frac{1}{5}, \frac{1}{3}\right)$ & 0.0784 \\
$c_{2}$ & $\left(\frac{1}{5}, \frac{1}{3}, 1\right)$ & 1 & $\left(\frac{1}{9}, \frac{1}{9}, \frac{1}{7}\right)$ & $\left(\frac{1}{7}, \frac{1}{5}, \frac{1}{3}\right)$ & $\left(\frac{1}{9}, \frac{1}{7}, \frac{1}{5}\right)$ & \\
$c_{3}$ & $(5,7,9)$ & $(7,9,9)$ & 1 & $(3,5,7)$ & $(1,3,5)$ & \\
$c_{4}$ & $(1,3,5)$ & $(3,5,7)$ & $\left(\frac{1}{7}, \frac{1}{5}, \frac{1}{3}\right)$ & 1 & $\left(\frac{1}{5}, \frac{1}{3}, 1\right)$ & \\
$c_{5}$ & $(3,5,7)$ & $(5,7,9)$ & $\left(\frac{1}{5}, \frac{1}{3}, 1\right)$ & $(1,3,5)$ & 1 & \\
\hline
\end{tabular}

Table 3: Fuzzy pairwise comparison matrix of the criteria.

\begin{tabular}{lcccccc}
\hline$c_{1}$ & Prague & $c p_{11}$ & $c p_{21}$ & $c p_{31}$ & fuzzy priorities & $C O A_{\widetilde{C R}}$ \\
\hline Prague & 1 & $(1,3,5)$ & $(5,7,9)$ & $(7,9,9)$ & $(0.4103,0.5962,0.6956)$ & 0.0867 \\
$c p_{11}$ & $\left(\frac{1}{5}, \frac{1}{3}, 1\right)$ & 1 & $(1,3,5)$ & $(5,7,9)$ & $(0.1513,0.2616,0.4447)$ & \\
$c p_{21}$ & $\left(\frac{1}{9}, \frac{1}{7}, \frac{1}{5}\right)$ & $\left(\frac{1}{5}, \frac{1}{3}, 1\right)$ & 1 & $(1,3,5)$ & $(0.0557,0.0989,0.1922)$ & \\
$c p_{31}$ & $\left(\frac{1}{9}, \frac{1}{9}, \frac{1}{7}\right)$ & $\left(\frac{1}{9}, \frac{1}{7}, \frac{1}{5}\right)$ & $\left(\frac{1}{5}, \frac{1}{3}, 1\right)$ & 1 & $(0.0318,0.0434,0.0821)$ & \\
\hline
\end{tabular}

Table 4: Fuzzy pairwise comparison matrix for $a_{1}=$ "Prague" according to criterion $c_{1}=$ "Price" and the derived fuzzy priorities.

The overall fuzzy priorities of the typical examples and the Prague package were obtained by the fuzzy weighted average of the fuzzy priorities from Tab. 4-8 with the weights derived from Tab. 3. They are presented in Tab. 9 and graphically illustrated in Figure 2. For these 


\begin{tabular}{lcccccc}
\hline$c_{2}$ & Prague & $c p_{12}$ & $c p_{22}$ & $c p_{32}$ & fuzzy priorities & $C O A_{\widetilde{C R}}$ \\
\hline Prague & 1 & $\left(\frac{1}{3}, 1,3\right)$ & $\left(\frac{1}{3}, 1,3\right)$ & $(5,7,9)$ & $(0.1484,0.3182,0.5424)$ & 0.0662 \\
$c p_{12}$ & $\left(\frac{1}{3}, 1,3\right)$ & 1 & $\left(\frac{1}{3}, 1,3\right)$ & $(5,7,9)$ & $(0.1484,0.3182,0.5424)$ & \\
$c p_{22}$ & $\left(\frac{1}{3}, 1,3\right)$ & $\left(\frac{1}{3}, 1,3\right)$ & 1 & $(5,7,9)$ & $(0.1484,0.3182,0.5424)$ & \\
$c p_{32}$ & $\left(\frac{1}{9}, \frac{1}{7}, \frac{1}{5}\right)$ & $\left(\frac{1}{9}, \frac{1}{7}, \frac{1}{5}\right)$ & $\left(\frac{1}{9}, \frac{1}{7}, \frac{1}{5}\right)$ & 1 & $(0.0325,0.0455,0.0625)$ & \\
\hline
\end{tabular}

Table 5: Fuzzy pairwise comparison matrix for $a_{1}=$ "Prague" according to criterion $c_{2}=$ "Quality of the accommodation" and the derived fuzzy priorities.

\begin{tabular}{lcccccc}
\hline$c_{3}$ & Prague & $c p_{13}$ & $c p_{23}$ & $c p_{33}$ & fuzzy priorities & $C O A_{\widetilde{C R}}$ \\
\hline Prague & 1 & $\left(\frac{1}{9}, \frac{1}{9}, \frac{1}{7}\right)$ & $\left(\frac{1}{7}, \frac{1}{5}, \frac{1}{3}\right)$ & $\left(\frac{1}{3}, 1,3\right)$ & $(0.0372,0.0572,0.1066)$ & 0.0994 \\
$c p_{13}$ & $(7,9,9)$ & 1 & $(3,5,7)$ & $(7,9,9)$ & $(0.5547,0.6642,0.7153)$ & \\
$c p_{23}$ & $(3,5,7)$ & $\left(\frac{1}{7}, \frac{1}{5}, \frac{1}{3}\right)$ & 1 & $(3,5,7)$ & $(0.1553,0.2214,0.3217)$ & \\
$c p_{33}$ & $\left(\frac{1}{3}, 1,3\right)$ & $\left(\frac{1}{9}, \frac{1}{9}, \frac{1}{7}\right)$ & $\left(\frac{1}{7}, \frac{1}{5}, \frac{1}{3}\right)$ & 1 & $(0.0372,0.0572,0.1066)$ & \\
\hline
\end{tabular}

Table 6: Fuzzy pairwise comparison matrix for $a_{1}=$ "Prague" according to criterion $c_{3}=$ "Location" and the derived fuzzy priorities.

\begin{tabular}{lcccccc}
\hline$c_{4}$ & Prague & $c p_{14}$ & $c p_{24}$ & $c p_{34}$ & fuzzy priorities & $C O A_{\widetilde{C R}}$ \\
\hline Prague & 1 & $\left(\frac{1}{7}, \frac{1}{5}, \frac{1}{3}\right)$ & $\left(\frac{1}{7}, \frac{1}{5}, \frac{1}{3}\right)$ & $\left(\frac{1}{3}, 1,3\right)$ & $(0.0444,0.0790,0.1530)$ & 0.0697 \\
$c p_{14}$ & $(3,5,7)$ & 1 & $\left(\frac{1}{3}, 1,3\right)$ & $(7,9,9)$ & $(0.2706,0.4576,0.6253)$ & \\
$c p_{24}$ & $(3,5,7)$ & $\left(\frac{1}{3}, 1,3\right)$ & 1 & $(3,5,7)$ & $(0.2204,0.3951,0.5853)$ & \\
$c p_{34}$ & $\left(\frac{1}{3}, 1,3\right)$ & $\left(\frac{1}{9}, \frac{1}{9}, \frac{1}{7}\right)$ & $\left(\frac{1}{7}, \frac{1}{5}, \frac{1}{3}\right)$ & 1 & $(0.0416,0.0682,0.1253)$ & \\
\hline
\end{tabular}

Table 7: Fuzzy pairwise comparison matrix for $a_{1}=$ "Prague" according to criterion $c_{4}=$ "Length" and the derived fuzzy priorities.

\begin{tabular}{lcccccc}
\hline$c_{5}$ & Prague & $c p_{15}$ & $c p_{25}$ & $c p_{35}$ & fuzzy priorities & $C O A_{\widetilde{C R}}$ \\
\hline Prague & 1 & $\left(\frac{1}{7}, \frac{1}{5}, \frac{1}{3}\right)$ & $\left(\frac{1}{3}, 1,3\right)$ & $(1,3,5)$ & $(0.0696,0.1456,0.2777)$ & 0.0918 \\
$c p_{15}$ & $(3,5,7)$ & 1 & $(3,5,7)$ & $(7,9,9)$ & $(0.5118,0.6405,0.7125)$ & \\
$c p_{25}$ & $\left(\frac{1}{3}, 1,3\right)$ & $\left(\frac{1}{7}, \frac{1}{5}, \frac{1}{3}\right)$ & 1 & $(3,5,7)$ & $(0.0918,0.1654,0.3013)$ & \\
$c p_{35}$ & $\left(\frac{1}{5}, \frac{1}{3}, 1\right)$ & $\left(\frac{1}{9}, \frac{1}{9}, \frac{1}{7}\right)$ & $\left(\frac{1}{7}, \frac{1}{5}, \frac{1}{3}\right)$ & 1 & $(0.0350,0.0485,0.0929)$ & \\
\hline
\end{tabular}

Table 8: Fuzzy pairwise comparison matrix for $a_{1}=$ "Prague" according to criterion $c_{5}=$ "Type of holiday" and the derived fuzzy priorities. 
overall fuzzy priorities, dissemblance indexes were calculated (Tab. 9 ). It results that the smallest dissemblance index is obtained for the overall fuzzy priority of the typical example of class $C_{3}$, therefore the Prague package belongs to class $C_{3}$.

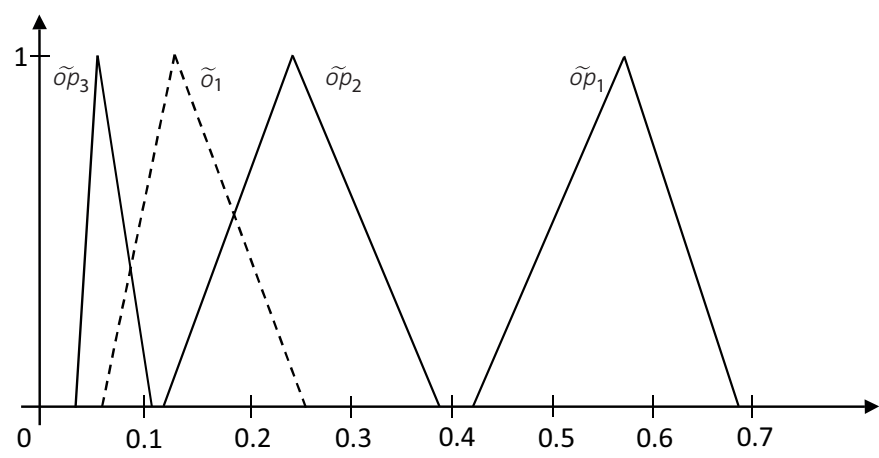

Figure 2: Overall fuzzy priorities of $a_{1}=$ "Prague" and of corresponding central profiles.

\begin{tabular}{rll}
\hline & Overall fuzzy priorities & Dissemblance index \\
\hline$\widetilde{o}_{1}=(0.0625,0.1262,0.2608)$ & \\
$\widetilde{o p}_{11}=(0.4259,0.5942,0.6884)$ & $D\left(\widetilde{o}_{1}, \widetilde{o p}_{11}\right)=0.8635$ \\
$\widetilde{o p}_{12}=(0.1256,0.2245,0.3833)$ & $D\left(\widetilde{o}_{1}, \widetilde{o p}_{12}\right)=0.1911$ \\
$\widetilde{o p}_{13}=(0.0364,0.0551,0.1048)$ & $D\left(\widetilde{o}_{1}, \widetilde{o p}_{13}\right)=0.1623$ \\
\hline
\end{tabular}

Table 9: Overall fuzzy priorities of $a_{1}=$ "Prague" and of corresponding central profiles and dissemblance indexes.

The same process is repeated for the other alternatives. Their assignments are given in Tab. 10. It can be seen that Bulgaria is the only alternative assigned to class $C_{1}$. It is, therefore, the holiday package that is recommended to the customer.

\section{Conclusion}

A fuzzy extension of the AHPSort method called FAHPSort has been proposed in this paper in order to deal with sorting decision-making 


\begin{tabular}{lc}
\hline Alternatives & Class \\
\hline$a_{1}=$ "Prague" & $C_{3}$ \\
$a_{2}=$ "Paris" & $C_{3}$ \\
$a_{3}=$ "Amsterdam" & $C_{2}$ \\
$a_{4}=$ "Croatia" & $C_{2}$ \\
$a_{5}=$ "Bulgaria" & $C_{1}$ \\
$a_{6}=$ "Mallorca" & $C_{2}$ \\
$a_{7}=$ "Norway" & $C_{3}$ \\
$a_{8}=$ "Switzerland" & $C_{3}$ \\
\hline
\end{tabular}

Table 10: Sorting of the alternatives into classes.

problems with vague or imprecise information by using pairwise comparison techniques, and its application was illustrated on a tourism decision-making problem. The FAHPSort method allows us to capture the imprecision of information in decision-making problems and the vagueness in meaning of the linguistic terms expressing the intensities of preference of one object over another by using triangular fuzzy numbers. Furthermore, the FAHPSort method proposed in this paper, unlike most fuzzy AHP methods proposed so far, properly reflects the reciprocity of pairwise comparisons of objects, which is the key property of the pairwise comparison matrices in AHP methods. The reciprocity property is preserved throughout the whole computation process, which allows us to obtain more precise and reliable results. Further, five important properties of sorting methods have been examined and proved to hold for the FAHPSort method - independence, homogeneity, uniqueness, monotonicity, and conformity.

The FAHPSort method can be easily adapted to decision-making problems where trapezoidal fuzzy numbers or any other type of fuzzy numbers described uniquely by their $\alpha$-cuts are used to model the imprecision of information or the vagueness in meaning of linguistic terms expressing the intensity of preference of one object over another.

Despite the desirable properties mentioned above, the FAHPSort method has also its drawback. Same as in the AHPSort method, decision makers may have difficulties to define central or limiting profiles for criteria with which they are not familiar. When the decision makers are unable to define the profiles, the FAHPSort method cannot be used and another method has to be applied.

The FAHPSort method introduced in this paper provides a lot of 
space for future research. For example, it would be interesting to perform sensitivity analysis in order to compare the two sorting rules for central profiles proposed in Section 3.4.2. Also, other aggregation methods may be applied instead of the fuzzy weighted average in order to deal with the compensation issue mentioned in Section 2.2. Finally, AHPSort could also be fuzzified by employing type-2 fuzzy sets or intuitionistic fuzzy sets. Consequently, the the performance of these other methods could be compared with the performance of the FAHPSort method based on triangular fuzzy numbers introduced in this paper.

\section{A}

$\mathbf{a}_{1}=$ Prague:

- price:212 €

- quality of accommodation: hotel **

- location: Prague

- length of the holiday: 5 days

- type of the holiday: sightseeing

$\mathbf{a}_{2}=$ Paris:

- price: $240 €$

- quality of accommodation: hotel **

- location: Paris

- length of the holiday: 5 days

- type of the holiday: sightseeing

$\mathbf{a}_{3}=$ Amsterdam:

- price: $329 €$

- quality of accommodation: hotel ***

- location: Amsterdam

- length of the holiday: 5 days

- type of the holiday: sightseeing

$\mathbf{a}_{4}=$ Croatia: 
- price: $243 €$

- quality of accommodation: hotel **

- location: seaside in Petrčane (near to Zadar, Adriatic Sea)

- length of the holiday: 8 days

- type of the holiday: relaxing in the seaside

$\mathbf{a}_{5}=$ Bulgaria:

- price: $326 €$

- quality of accommodation: hotel $* * *$

- location: seaside in Burgas (Black Sea)

- length of the holiday: 7 days

- type of the holiday: relaxing in the seaside

$\mathbf{a}_{6}=$ Mallorca

- price: $431 €$

- quality of accommodation: hotel ***

- location: seaside in Bay of Alcudia

- length of the holiday: 8 days

- type of the holiday: relaxing in the seaside

$\mathbf{a}_{7}=$ Norway:

- price: $831 €$ - the price includes also the cruise

- quality of accommodation: *** rooms on the excursion boat

- location: cruise from Copenhagen to fjords of Norway

- length of the holiday: 8 days

- type of the holiday: visiting natural environment

$\mathbf{a}_{8}=$ Switzerland:

- price: $1380 €$ - the price includes also transport during the tour

- quality of accommodation: hotel ***

- location: tour in Switzerland comprising of the tour from Genova to Motreux, lake cruise to Lausanne, The Goldeen Pass Line, Interlaken, Jungfraujoch (The Ice Cave), visit of Luzern, travel to Engelberg by the world's first cabel car with 360degree view

- length of the holiday: 6 days

- type of the holiday: visiting natural environment and sightseeing 


\section{References}

1 K. T. Atanassov. Intuitionistic fuzzy sets. Fuzzy Sets and Systems, 20:87-96, 1986.

2 F. Bronner and R. de Hoog. Agreement and disagreement in family vacation decision-making. Tourism Management, 29:967-979, 2008.

3 J. J. Buckley. Fuzzy hierarchical analysis. Fuzzy Sets and Systems, 17:233-247, 1985.

4 J. J. Buckley, T. Feuring, and Y. Hayashi. Fuzzy hierarchical analysis revised. European Journal of Operational Research, 129:48-64, 2001.

5 A. Calabrese, R. Costa, and T. Menichini. Using fuzzy ahp to manage intellectual capital assets: An application to the ict service industry. Expert Systems with Applications, 40:3747-3755, 2013.

6 D. Y. Chang. Applications of the extent analysis method on fuzzy ahp. European Journal of Operational Research, 95:649-655, 1996.

7 C. H. Cheng. Evaluating naval tactical missile systems by fuzzy ahp based on the grade value membership fuction. European Journal of Operational Research, 96:343-355, 1997.

8 C. H. Cheng and D. L. Mon. Evaluating weapon system by analytical hierarchy process based on fuzzy scales. Fuzzy Sets and Systems, 63:1-10, 1994.

9 F. Chiclana, E. Herrera-Viedma, S. Alonso, and F. Herrera. Cardinal consistency of reciprocal preference relations: A characterization of multiplicative transitivity. IEEE Transactions on Fuzzy Systems, 17:14-23, 2009.

10 A. T. W. Chu, R. E. Kalaba, and K. Spingarn. A comparison of two methods for determining the weights of belonging to fuzzy sets. Journal of Optimization Theory and Applications, 27(1):531-538, 1979.

11 C. Crawford and C. A. Williams. A note on the analysis of subjective judgment matrices. Journal of Mathematical Psychology, 29:387-405, 1985.

12 R. de F. S. M. Russo and R. Camanho. Criteria in ahp: a systematic review of literature. Information Technology and Quantitative management, 55(1):1123-1132, 2015. 
13 M. Enea and T. Piazza. Project selection by constrained fuzzy ahp. Fuzzy Optimization and Decision Making, 3:39-62, 2004.

14 M. Fedrizzi and J. Krejčí. A note on the paper "fuzzy analytic hierarchy process: Fallacy of the popular methods". International Journal of Uncertainty, Fuzziness and Knowledge-Based Systems, 23:965-970, 2015.

15 R. K. Goyal and S. Kaushal. Deriving crisp and consistent priorities for fuzzy ahp-based multicriteria systems using non-linear constrained optimization. Fuzzy Optimization and Decision Making, pages 1-15, 2017.

16 G. Gujansky and M. C. N. Belderrain. Ahpsort method applied to an automobile acquisition. Revista Gestão em Engenharia, 1(1):1$17,2014$.

17 E. Herrera-Viedma, F. Herrera, F. Chiclana, and M. Luque. Some issues on consistency of fuzzy preference relations. European Journal of Operational Research, 3:98-109, 2004.

18 W. Ho. Integrated analytic hierarchy process and its applications - a literature review. European Journal of Operational Research, 186(1):211-228, 2008.

19 W. Ho and X. Ma. The state-of-the-art integrations and applications of the analytic hierarchy process. European Journal of Operational Research, 267(1):399-414, 2018.

20 A. Ishizaka and N. H. Nguyen. Calibrated fuzzy ahp for current bank selection. Expert Systems with Applications, 40:3775-3783, 2013.

21 A. Ishizaka, C. Pearman, and P. Nemery. Ahpsort: an ahp-based method for sorting problems. International Journal of Production research, 50:4767-4784, 2012.

22 A. Kaufman and M. M. Gupta. Introduction to fuzzy arithmetic: Theory and Applications. Van Nostrand Reinhold Co., New York, 1985.

23 B. Kirubakaran and M. Ilangkumaran. Selection of optimum maintenance strategy based on fahp integrated with gra-topsis. Annals of Operations Research, pages 1-29, 2015.

24 G. J. Klir and B. Yuan. Constrained fuzzy arithmetic: Basic questions and some answers. Soft Computing, 2:100-108, 1998. 
25 G. Kou, D. Ergu, C. Lin, and Y. Chen. Pairwise comparison matrix in multiple criteria decision making. Technological and Economic Development of Economy, 22(5):738-765, 2016.

26 G. Kou and C. Lin. A cosine maximization method for the priority vector derivation in ahp. European Journal of Operational Research, 235(1):225-232, 2014.

27 G. Kou and J. Shang. Enhancing data consistency in decision matrix: Adapting hadamard model to mitigate judgment contradiction. European Journal of Operational Research, 236(1):261-271, 2014.

28 J. Krejčí. Fuzzy eigenvector method for deriving normalized fuzzy priorities from fuzzy multiplicative pairwise comparison matrices. Fuzzy Optimization and Decision Making, submitted, 2017.

29 J. Krejčí. Fuzzy eigenvector method for obtaining normalized fuzzy weights from fuzzy pairwise comparison matrices. Fuzzy Sets and Systems, 315(1):26-43, 2017.

30 J. Krejčí. Pairwise comparison matrices and their fuzzy extension: Multi-criteria decision making with a new fuzzy approach. in book series Studies in Fuzziness and Soft Computing, 366, 2018.

31 J. Krejčí, O. Pavlačka, and J. Talašová. A fuzzy extension of analytic hierarchy process based on the constrained fuzzy arithmetic. Fuzzy Optimization and Decision Making, 16:89-110, 2017.

32 J. Krejčí and J. Stoklasa. Fuzzified ahp in the evaluation of scientific monographs. Central European Journal of Operations Research, 24:353-370, 2015.

33 J. Krejčí and J. Talašová. A proper fuzzification of saaty's scale and an improved method for computing fuzzy weights in fuzzy ahp. Proceedings of the 31th International Conference on Mathematical Methods in Economics 2013, Jihlava, ISBN: 978-80-8703576-4:452-457, 2013.

34 V. B. Kreng and C. Y. Wu. Evaluation of knowledge portal development tools using a fuzzy ahp approach: The case of taiwanese stone industry. European Journal of Operational Research, 176:1795-1810, 2007.

35 S. Kubler, A. Voisin, W. Derigent, A. Thomas, E. Rondeau, and K. Främling. Group fuzzy ahp approach to embed relevant data on "communicating material". Computers in Industry, 65:675-692, 2014 . 
36 P. J. M. V. Laarhoven and M. Pedrycz. A fuzzy extension of saaty's priority theory. Fuzzy Sets and Systems, 11:199-227, 1983.

37 M. J. Liberatore and R. L. Nydick. The analytic hierarchy process in medical and health care decision making: A literature review. European Journal of Operational Research, 189(1):194-207, 2008.

38 C. López and A. Ishizaka. Gahpsort: A new group multi-criteria decision method for sorting a large number of the cloud-based erp solutions. Computes in Industry, 92-93(1):12-24, 2017.

39 F. Miccoli and A. Ishizaka. Sorting municipalities in umbria according to the risk of wolf attacks with ahpsort ii. Ecological Indicators, 73(1):741-755, 2017.

40 L. Mikhailov. Deriving priorities from fuzzy pairwise comparison judgments. Fuzzy Sets and Systems, 134:365-385, 2003.

41 D. Ozgen and B. Gulsun. Combining possibilistic linear programming and fuzzy ahp for solving the multi-objective capacitated multi-facility location problem. Information Sciences, 268:185201, 2014.

42 J. Rezaei and R. Ortt. Multi-criteri segmentation using a fuzzy preference relations based ahp. European Journal of Operational Research, 225:75-84, 2013.

43 R. W. Saaty. The analytic hierarchy process - what it is and how it is used. Mathematical Modelling, 9:161-176, 1987.

44 T. L. Saaty. The analytic hierarchy process. McGraw Hill, New York, 1980.

45 N. Somsuk and T. Laosirihongthong. A fuzzy ahp to prioritize enabling factors for strategic management of university business incubators: Resource-based view. Technological Forecasting and Social Change, 85:198-210, 2014.

46 Z. Song, H. Zhu, G. Jia, and C. He. Comprehensive evaluation on self-ignition risks of coal stockpiles using fuzzy ahp approaches. Journal of Loss Prevention in the Process Industries, 32:78-94, 2014.

47 J. Stoklasa, V. Jandová, and J. Talašová. Weak consistency in saaty's ahp - evaluating creative work outcomes of czech art colleges. Neural Network World, 23:61-77, 2013. 
48 N. Subramanian and R. Ramanathan. A review of applications of analytic hierarchy process in operations management. International Journal of Production Economics, 128(1):215-241, 2012.

49 T. Takagi and M. Sugeno. Fuzzy identification of systems and its application to modeling and control. IEEE Transitions on Systems, Man, and Cybernetics, 15:116-132, 1985.

50 S. Tesfamariam and R. Sadiq. Risk-based environmental decisionmaking using fuzzy analytic hierarchy process (f-ahp). Stochastic Environmental Research and Risk Assessment, 21:35-50, 2006.

51 O. S. Vaidya and S. Kumar. Analytic hierarchy process: An overview of applications. European Journal of Operational Research, 169(1):1-29, 2006.

52 C.-K. S. Wong and W.-Y. Y. Kwong. Outbound tourist' selection criteria for choosing all-inclusive package tours. Tourism Management, 25:581-592, 2004.

53 R. Xu. Fuzzy least-square priority method in the analytic hierarchy process. Fuzzy Sets and Systems, 112:395-404, 2000.

54 L. A. Zadeh. The concept of a linguistic variable and its application to approximate reasoning - i. Information Sciences, 8:199-249, 1975 .

55 K. Zhü. Fuzzy analytic hierarchy process: Fallacy of the popular methods. European Journal of Operational Research, 236:209-214, 2014 . 